\title{
Acute Rheumatic Myocarditis
}

National Cancer Institute

\section{Source}

National Cancer Institute. Acute Rheumatic Myocarditis. NCI Thesaurus. Code C35202.

Inflammation of the myocardium in acute rheumatic heart disease. 